\title{
Serum levels of retinol-binding protein 4 and risk of colon adenoma
}

\section{Dear Editor,}

Obesity is now considered as a convincing and probable cause for colorectal neoplasia (World Cancer Research Fund 2011). Insulin resistance is a key mechanism relating obesity to colon carcinogenesis (Giovannucci 2001). Supporting evidence for this notion includes data from a number of epidemiological studies demonstrating the risk association of colon cancer and adenoma with insulin resistance-associated biomarkers, such as insulin, insulinlike growth factors, adipokines (e.g. leptin and adiponectin), and inflammatory cytokines (e.g. IL6 and TNF $\alpha$ ) (Giovannucci 2001, Sandhu et al. 2002, Ortiz et al. 2012).

Retinol-binding protein 4 (RBP4), a recently identified adipokine, has been shown to potentially have a causative effect on the development of insulin resistance (Yang et al. 2005, Graham et al. 2006). RBP4 increases expression of the glucose transporter, GLUT4 (SLC2A4), which facilitates the rate-limiting step in glucose uptake by skeletal muscle and adipocytes. In adipose tissue-specific Glut4 knockout (adipose-Glut4 ${ }^{-/-}$) mice, secretion of RBP4 by adipocytes is greatly enhanced. Increased circulating levels of RBP4 suppress phosphoinositide-3-kinase in muscle cells, and in the liver, stimulate the expression of phosphoenolpyruvate carboxykinase (PEPCK), inducing systemic insulin resistance. Treatment of adipose-Glut $4^{-/-}$ mice with rosiglitazone, an insulin sensitizer, reduced the elevated RBP4 mRNA levels in adipose tissue and completely normalized the serum RBP4 levels (Yang et al. 2005). In humans, subcutaneous adipocyte GLUT4 protein levels correlated positively with the rate of glucose disposal and negatively with serum levels of RBP4 (Graham et al. 2006).

Despite the emerging evidence for a causal relationship of RBP4 to insulin resistance, no previous study has investigated the relationship between RBP4 and colon neoplasia. Therefore, we carried out a cross-sectional study to examine the hypothesis that high levels of RBP4 are associated with an increased risk of colon adenoma.

\section{Materials and methods}

The study population consisted of 626 patients who were referred for colonoscopy screening at the University Hospitals Health System (UHHS) from January 2006 to March 2007. Out of them, 196 patients who had histologically confirmed adenomatous polyps in the colon were defined as cases, and the remaining 430 patients with negative screening were defined as controls. We excluded those with the following indications: i) a prior diagnosis of any cancer, colorectal adenoma, or inflammatory bowel diseases; ii) a known family history of hereditary non-polyposis colon cancer, familial adenomatous polyps, or other cancer syndromes; and iii) below the age of 30 . Of the eligible participants, $64.9 \%$ agreed to participate.

Each participant completed a Computer-Assisted Personal Interview (CAPI) based on the Risk Factor Questionnaire (RFQ) developed by the NCI Colon Cancer Family Registry (http://epi.grants.cancer.gov/CFR/about_ questionnaires.html). A research nurse obtained measurements of height, weight, and fasting blood samples from each participant just before their colonoscopy examination.

Blood samples were immediately processed in a research laboratory and stored at $-80^{\circ} \mathrm{C}$. Serum RBP4 levels were measured by ELISAs (Alpco Diagnostics, Salem, $\mathrm{NH}$, USA). Laboratory personnel were blind to the case and control statuses of each participant and RBP4 assays were performed twice, the average of which were used for the final value for each participant. Serum levels of insulin and glucose were available for 593 patients, and the homeostasis model assessment of insulin resistance (HOMA-IR) was calculated (glucose $\times$ insulin/405). Of the 33 patients for whom HOMA-IR calculations were missing, 17 were controls and 16 were cases, and there was no statistically significant difference in age, sex, and race between those with and without HOMA-IR data. All patients signed written consents and this study was approved by the University Hospitals Case Medical Center Institutional Review Board.

Published by Bioscientifica Ltd. 
We first performed univariate analyses of BMI, serum RBP4, and other covariates comparing the cases with the controls, using either ANOVA (for continuous variables) or a $\chi^{2}$ test (for discrete variables). We then applied unconditional logistic regression to estimate coefficients and $95 \%$ CIs for the association between $\log$ (RBP4) and colon adenomas. In our base model, we adjusted for age, race, and sex. We then further adjusted for BMI, family history of colorectal cancer, smoking, and NSAID use (full model). We also compared $\log (\mathrm{RBP} 4)$ results from multivariate logistic regression models with and without adjustment for HOMA (full model+HOMA). Odds ratio (OR) estimates for continuous scale were calculated for each $35 \mu \mathrm{g} / \mathrm{ml}$ increment ( $\sim$ s.D.) in RBP4 levels.

To examine the potential modifying effect of BMI, we categorized each individual into 'lower' or 'higher' BMI group based on the median value $\left(27.8 \mathrm{~kg} / \mathrm{m}^{2}\right)$. RBP4 was then analyzed as a categorical variable based on the tertile cut-off values of the entire study population. Categorical RBP4 was analyzed for both 'lower' and 'higher' BMI groups in all three models (base model, full model, and full model + HOMA). Tests for trend were based on logistic regression coefficients for continuous scale. We tested for multiplicative interaction between RBP4 and BMI by including their cross-product term in the models. All $P$ values were two-sided and computed using R i386 3.0.2.

\section{Results}

The cases had higher levels of RBP4 than the controls $($ mean $=58.5 \mu \mathrm{g} / \mathrm{ml} \pm 38.2$ vs $51.9 \mu \mathrm{g} / \mathrm{ml} \pm 32.5 ; P=0.03$ ). Smoking, sex, and age differed significantly between the cases and the controls as well (Supplementary Table 1, see section on supplementary data given at the end of this article). In base model, $\log$ (RBP4) was in statistically significant association with the risk of colon adenoma $(\mathrm{OR}=3.10, \mathrm{CI}=1.15-8.66 ; P=0.03)$. Further adjustment for BMI, NSAIDs, family history of colorectal cancer, diabetes mellitus (DM), and smoking (full model) had little impact on the results. Adding HOMA-IR to the full model yielded similar results. As treatment of DM may reduce RBP4 levels, we then repeated the analyses excluding 90 patients with DM. The estimates were virtually identical to the analyses based on the entire sample, except for a slight decrease in $\beta$-slope in HOMA-IR adjusted model (Table 1).

To explore potential effect modification by obesity, we performed analyses stratified by BMI $\left(<27.8 \mathrm{~kg} / \mathrm{m}^{2}\right.$ (median) vs $\geq 27.8 \mathrm{~kg} / \mathrm{m}^{2}$ ). For those with a lower BMI, there is an approximately twofold increase in the adenoma risk for the top tertile $(\mathrm{OR}=2.07, \mathrm{CI}=1.04$ 4.11; $\left.P_{\text {trend }}=0.04\right)$; in contrast, the corresponding OR estimate was $1.02(\mathrm{CI}=0.53-1.97)$ for those with a higher BMI $\left(P_{\text {trend }}=0.93\right)$. Excluding patients with DM revealed an even stronger association among those with a lower BMI (Fig. 1). Again, there is little evidence for the association among those with a higher BMI. Further adjustment of HOMA-IR yielded similar results (data not shown). Tests for multiplicative interaction between RBP4 and BMI groups were not statistically significant $(P>0.10)$.

\section{Discussion}

This is the first population-based study relating circulating levels of RBP4 to the risk of colon neoplasia. The mechanisms by which RBP4 may contribute to colon carcinogenesis are largely unknown. Our analysis was motivated by the discovery of RBP4 as a novel adipokine with a potentially causative role in insulin resistance (Yang et al. 2005, Graham et al. 2006). While our data indeed indicate a strong positive association, especially among patients with a lower BMI, the observed association is independent of insulin resistance, suggesting that mechanisms other than insulin resistance may underlie

Table 1 Logistic regression analysis of risk of adenoma for serum levels of RBP4

\begin{tabular}{|c|c|c|c|c|}
\hline & \multicolumn{4}{|c|}{ Including DM patients ${ }^{a}$} \\
\hline & $\beta \pm$ S.E.M. & $\mathrm{OR}^{\mathrm{b}}$ & $95 \% \mathrm{Cl}$ & $P$ \\
\hline Base model ${ }^{\mathrm{C}}$ & $0.32 \pm 0.15$ & 3.1 & $1.15-8.66$ & 0.03 \\
\hline Full model $^{\mathrm{d}}$ & $0.32 \pm 0.15$ & 3.1 & $1.11-8.97$ & 0.03 \\
\hline Full model + HOMA & $0.32 \pm 0.16$ & 3.1 & $1.04-8.97$ & 0.04 \\
\hline
\end{tabular}

\begin{tabular}{|c|c|c|c|}
\hline \multicolumn{4}{|c|}{ Excluding DM patients } \\
\hline$\beta \pm$ S.E.M. & $\mathrm{OR}^{\mathrm{b}}$ & $95 \% \mathrm{Cl}$ & $P$ \\
\hline $0.32 \pm 0.16$ & 3.13 & $0.01-9.49$ & 0.04 \\
\hline $0.32 \pm 0.16$ & 3.13 & $1.004-9.84$ & 0.04 \\
\hline $0.29 \pm 0.17$ & 2.82 & $0.87-8.84$ & 0.09 \\
\hline
\end{tabular}

${ }^{a}$ Diabetes treatment status is included in the three models as a covariate.

${ }^{b}$ ORs for each $35 \mu \mathrm{g} / \mathrm{ml}$ ( $\sim 1$ s.D.) increment in RBP4 levels.

'Base model adjustments for age, race, and sex.

${ }^{\mathrm{d} F u l l}$ model adjustments for age, race, sex, BMI, non-steroidal anti-inflammatory drug use (6 months or longer), known family history, and smoking (pack years).

e33 missing values in HOMA-IR index. 

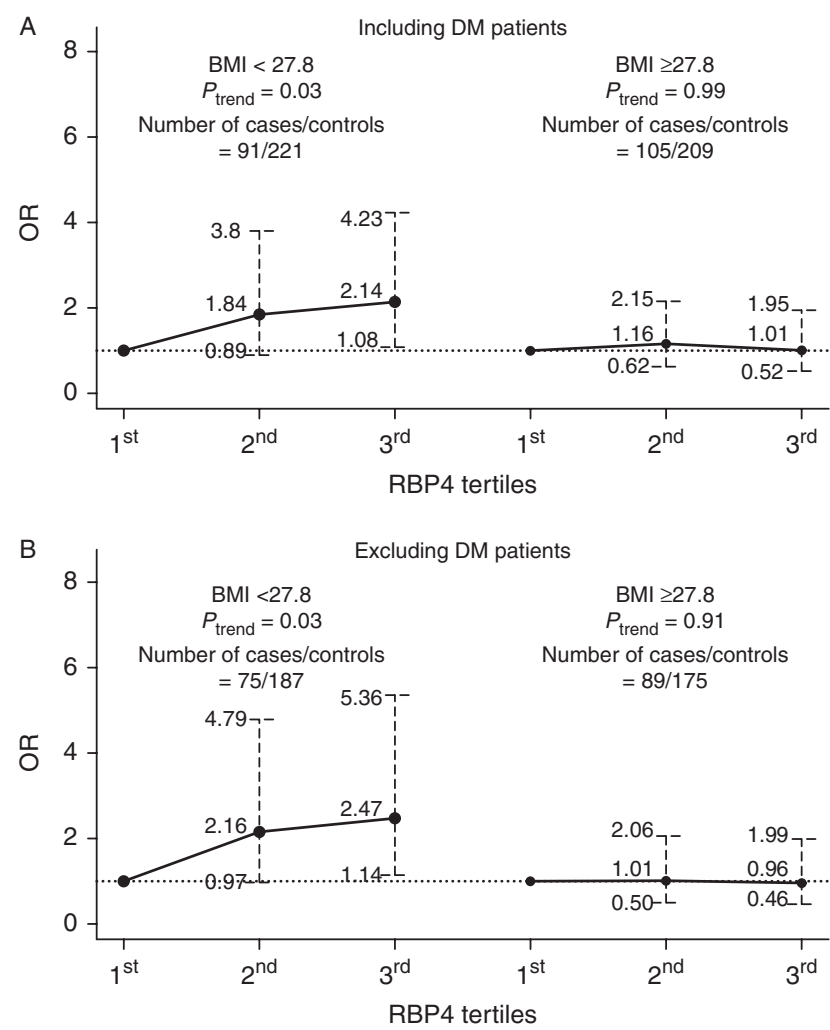

Figure 1

OR's for colon adenoma by RBP4. (A) Full model estimates based on entire sample of 626 patients. DM is included in the model as a covariate. (B) Full model excluding 90 patients with DM.

the RBP4-colon neoplasia relationship. RBP4 is the principal transporter for retinol and a recent study has demonstrated that the holo-RBP protein may act through its own receptor STRA6, which transduces JAK2/STAT3 signaling, to initiate carcinogenic transformations (Berry et al. 2014). It is thus tempting to speculate that RBP4 may promote colon carcinogenesis through this pathway.

Our observation that the RBP4-adenoma association is limited to patients with a lower BMI is intriguing. Adipose tissue is recognized as an active endocrine organ secreting a number of bioactive adipokines, which regulate physiological and pathological processes such as insulin sensitivity and resistance, inflammation, immunity, and carcinogenesis (Kahn \& Flier 2000, Van Kruijsdijk et al. 2009). We speculate that the wideranging effects of adipose tissue dysfunction in the state of obesity are so pervasive that the relative contribution of RBP4 is diminished.

There are limitations of our study. First, the crosssectional nature of our study cannot readily dismiss reverse causality, i.e. the presence of adenoma may have elevated the circulating levels of RBP4. Secondly, although we found striking difference in risk association between the 'higher' and the 'lower' BMI groups, our study with a moderate sample size has limited statistical power to detect multiplicative interaction between BMI and RBP4; as such, chance finding remains a potential explanation of the observed differential association between the two BMI groups. Finally, there were considerable differences in smoking, sex, and age between the cases and controls. Although we have included these variables as covariates in our models, we recognize that statistical adjustment of smoking, sex, and age may not adequately control for confounding by these variables or other unmeasured factors.

In summary, we demonstrate that higher circulating levels of RBP4 are associated, independent of insulin resistance and $\mathrm{DM}$, with the risk of colon adenoma, in particular among those with a lower BMI $\left(<27.8 \mathrm{~kg} / \mathrm{m}^{2}\right)$. Further investigation is merited to corroborate our findings and unravel the underlying molecular mechanisms of the association between RBP4 and colon adenomas.

Matthew V Abola ${ }^{1}$ Cheryl L Thompson ${ }^{1,2,3}$ Zhengyi Chen ${ }^{1}$ Amitabh Chak ${ }^{4}$ Nathan A Berger ${ }^{3,4}$ John P Kirwan ${ }^{5}$ $\mathrm{Li} \mathrm{Li}^{1,2,3}$

${ }^{1}$ Department of Family Medicine and Community Health, Research Division, Case Western Reserve University, 11001 Cedar Avenue, Suite 200, Cleveland, Ohio 44016, USA

${ }^{2}$ Epidemiology and Biostatistics, Case Western Reserve University, 10900 Euclid Avenue, Cleveland, Ohio 44106, USA ${ }^{3}$ Transdisciplinary Research on Energetics and Cancer Program, Case Comprehensive Cancer Center, Case Western Reserve University and University Hospitals of Cleveland, 11100 Euclid Avenue, Cleveland, Ohio 44106, USA

${ }^{4}$ Department of Medicine, University Hospitals Case Medical Center, 11100 Euclid Avenue, Cleveland, Ohio 44106, USA

${ }^{5}$ Department of Pathobiology, Lerner Research Institute, Cleveland Clinic, 9500 Euclid Avenue, Cleveland,

Ohio 44195, USA

(Correspondence should be addressed to $L \mathrm{Li}$, Departments of Family Medicine and Community Health, Case Western Reserve University, 11000 Cedar Avenue, Suite 402, Cleveland, Ohio 44106-7136, USA; Email: lxl62@case.edu)

Published by Bioscientifica Ltd 


\section{Supplementary data}

This is linked to the online version of the paper at http://dx.doi.org/10.1530/ ERC-14-0429.

\section{Declaration of interest}

The authors declare that there is no conflict of interest that could be perceived as prejudicing the impartiality of the research reported.

\section{Funding}

This work was supported by the National Cancer Institute grants (R01CA136726 and U01CA181770), Case Center for Transdisciplinary Research on Energetics and Cancer (U54 CA 116867), and Case GI SPORE (P50CA50964).

\section{References}

Berry DC, Levi L \& Noy N 2014 Holo-retinol-binding protein and its receptor STRA6 drive oncogenic transformation. Cancer Research $\mathbf{7 4}$ 1-11. (doi:10.1158/0008-5472.CAN-14-1052)

Giovannucci E 2001 Insulin, insulin-like growth factors and colon cancer: a review of the evidence. Journal of Nutrition 131 3109S-3120S.
Graham TE, Yang Q, Blüher M, Hammarstedt A, Ciaraldi TP, Henry RR, Wason CJ, Oberbach A, Jansson P-A, Smith U et al. 2006 Retinolbinding protein 4 and insulin resistance in lean, obese, and diabetic subjects. New England Journal of Medicine 354 2552-2563. (doi:10.1056/ NEJMoa054862)

Kahn BB \& Flier JS 2000 Obesity and insulin resistance. Journal of Clinical Investigation 106 473-481. (doi:10.1172/JCI10842)

Ortiz AP, Thompson CL, Chak A, Berger NA \& Li L 2012 Insulin resistance, central obesity, and risk of colorectal adenomas. Cancer 118 1774-1781. (doi:10.1002/cncr.26454)

Sandhu MS, Dunger DB \& Giovannucci EL 2002 Insulin, insulin-like growth factor-I (IGF-I), IGF binding proteins, their biologic interactions, and colorectal cancer. Journal of the National Cancer Institute 94 972-980. (doi:10.1093/jnci/94.13.972)

Van Kruijsdijk RCM, van der Wall E \& Visseren FLJ 2009 Obesity and cancer: the role of dysfunctional adipose tissue. Cancer Epidemiology, Biomarkers \& Prevention 18 2569-2578. (doi:10.1158/1055-9965. EPI-09-0372)

World Cancer Research Fund \& American Institute for Cancer Research 2011 In Continuous Update Project Report. Food, Nutrition, Physical Activity, and the Prevention of Colorectal Cancer. London, UK: WCRF/AICR. (available at: http://www.dietandcancerreport.org/ cancer_resource_center/downloads/cu/Colorectal-Cancer-2011Report.pdf)

Yang Q, Graham TE, Mody N, Preitner F, Peroni OD, Zabolotny JM, Kotani K, Quadro L \& Kahn BB 2005 Serum retinol binding protein 4 contributes to insulin resistance in obesity and type 2 diabetes. Nature 436 356-362. (doi:10.1038/nature03711)

Received in final form 1 January 2015

Accepted 5 January 2015
(C) 2015 Society for Endocrinology Printed in Great Britain 